\title{
Violencia doméstica en Veracruz. ¿Pandemia silente?
}

\section{Domestic violence in Veracruz. Double pandemic?}

\section{Estela Casados González}

a Observatorio Universitario de Violencias contra las Mujeres, Facultad de Antropología, Universidad Veracruzana.

Contacto: ecasados@uv.mx

Recibido: 12 de agosto de 2020

Aceptado: 12 de octubre de 2020

RESUMEN: La violencia en el ámbito doméstico en México se encuentra ampliamente extendida e incuba diversos tipos de violencia de carácter psicológico, físico, sexual, económico, patrimonial y feminicida. Este entorno ya ha sido explorado como un posible escenario que merma la calidad de vida a sus integrantes, principalmente a las mujeres. En eventos de carácter extraordinario tales como la contingencia sanitaria derivada del SARSCoV-2, se recrudece este fenómeno social arraigado fuertemente en nuestro país. Este texto presenta algunas reflexiones derivadas de información oficial que documenta las lesiones y eventos violentos que tienen lugar en los hogares del país, los registros sobre el tema generados por organizaciones de la sociedad civil, así como lo acontecido en Veracruz durante el primer semestre de 2020 recabado por el Observatorio Universitario de Violencias contra las Mujeres.

Palabras clave: Violencia; mujeres; violencia doméstica; pandemia; Veracruz.

ABSTRACT: Domestic violence in Mexico is widely extended and it incubates various types of violence of psychological, physical, sexual, economic, patrimonial and femicidal nature. This environment has already been explored as a possible scenario that decreases the quality of life of its members, particularly women. During abnormal situations, such as the health contingency derived from SARS-CoV-2, this deeply rooted social phenomenon worsens in our country. This paper presents some reflections derived from official information that documents the injuries and violence that takes place in the country's homes, archived by civic society organizations and by the University's Observatory of Violence against Women, throughout the first semester of 2020 in Veracruz, 
Keywords: Violence; Women; Domestic Violence; Pandemic; Veracruz.

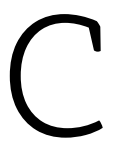

omo parte de las acciones para salvaguardar la vida de la población ante los efectos demoledores del SARS-CoV-2, en México el confinamiento de las familias que componen los casi 35 millones de hogares del país fue anunciado por el gobierno federal el 14 de marzo de 2020 y comenzó a realizarse gradualmente a partir del 23 de marzo de ese año. Poco después de tomarse estas medidas urgentes para enfrentar la letalidad del virus, diversas organizaciones civiles que trabajan a favor de los derechos humanos de las mujeres dieron voz de alerta: el confinamiento había puesto a millones de mexicanas a merced de la violencia que ya venían padeciendo por parte de algunos integrantes de sus familias.

Para comprender la magnitud de esta situación en México más allá de la contingencia sanitaria que actualmente vivimos en el mundo, es necesario destacar que la violencia contra las mujeres tiene su propio ritmo fatal, su propia trayectoria. La violencia contra las mujeres y concretamente la que tiene lugar en el ámbito doméstico, forma parte de un fenómeno más antiguo que el COVID - 19, pero es gracias a él que se ha recrudecido a la vez que ha cobrado mayor visibilidad.

En las siguientes páginas se presentan y analizan algunos datos sobre la violencia hacia las mujeres en el país previos a la contingencia sanitaria, con énfasis en las dinámicas que tienen lugar en el ámbito doméstico. Asimismo, se exponen los datos generados por instancias de los tres niveles de gobierno, organizaciones civiles a favor de los derechos humanos de las mujeres y aquellos que hemos generado desde el Observatorio Universitario de Violencias contra las Mujeres (OUVMujeres). ${ }^{1}$

\section{¿De qué hablamos cuando hablamos de violencia contra las mujeres?}

Si bien encontramos registros oficiales que datan de las dos últimas décadas del siglo XX, cuando hablamos sobre violencia contra las mujeres la información luce incompleta porque, por ejemplo, documenta defunciones femeninas con presunción de homicidio, pero deja de lado la violencia sistemática a la que están expuestas las mujeres a lo largo de su vida y en

\footnotetext{
${ }^{1}$ El Observatorio Universitario de Violencias contra las Mujeres pertenece a la Red de Observatorios de la Universidad Veracruzana, y se encuentra bajo el cuidado de la Coordinación Universitaria de Observatorios (CUO). Inició actividades en 2017 y fue impulsado desde la Licenciatura en Antropología Social de la Facultad de Antropología de la Universidad Veracruzana (FAUV). Su objetivo es visibilizar las violencias contra las mujeres en el estado de Veracruz a partir del monitoreo, sistematización y análisis de la información generada por medios de comunicación locales. A lo largo de este artículo, se hará referencia sobre él como OUVMujeres u Observatorio.
} 


\section{Artículos Científicos - Observatorio Universitario de Violencias contra las Mujeres}

momentos coyunturales de su existencia. Entonces, ¿de qué hablamos cuando nos referimos a la violencia contra las mujeres?

... la violencia de género contra las mujeres se sustenta en un conjunto de condiciones sociales, políticas, culturales, económicas e ideológicas que logran articular los procesos macrosociales con la vivencia de la subordinación en el orden de las relaciones cotidianas y en las escalas microsociales, lo que hace que cada mujer perciba su situación como algo personal, individual, no compartido con otras y mucho menos producto de una compleja construcción sociohistórica de poder y dominación (Castañeda, Ravelo y Pérez, 2013: p. 14

Retomando esta definición elaborada por Patricia Castañeda, Patricia Ravelo y Teresa Pérez, observamos que existe una confluencia de procesos macro y microsociales que permean la experiencia vivida de las mujeres en torno a la violencia. Tanto en lo cotidiano y en el marco de las relaciones cercanas como en las condiciones estructurales que definen las relaciones entre los géneros y el impacto de éstas en las mujeres, encontramos a la violencia como un elemento omnipresente en la definición de las identidades femeninas y masculinas.

Incluso, este conjunto de condiciones que refieren las autoras nos lleva hacia otras instancias que regulan las relaciones de género y también la violencia que se desencadena de ellas. Es decir, nos llevan hacia el Estado. (ibid) Como gran ente regulador, hace parte de las estrategias más letales de violencia contra las mujeres.

Retomando esta línea argumentativa, la antropóloga feminista Marcela Lagarde señala que existen condiciones para que el feminicidio se presente con mayor fuerza cuando el Estado no otorga garantías a las mujeres para su seguridad y preservación de sus vidas en la comunidad, hogar, espacio laboral, de tránsito o de esparcimiento: “cuando el Estado es parte estructural del problema por su signo patriarcal y por su preservación de dicho orden, el feminicidio es un crimen de Estado" (Lagarde, 2008: pp. 216-217).

Este crimen de Estado engarza con la desigualdad estructural entre mujeres y hombres, con la dominación de los hombres sobre las mujeres y la violencia de género a manera de mecanismo de reproducción de la opresión de las mujeres.

Marcela Lagarde también establece que:

De esas condiciones estructurales surgen otras condiciones culturales como son el ambiente ideológico y social de machismo y misoginia, y de normalización de la violencia contra las mujeres. Se suman también, ausencias legales y de políticas democráticas con contenido de género del gobierno y de los órganos de justicia del Estado, lo que produce impunidad y genera más injusticia, así como condiciones de convivencia insegura, pone en riesgo su vida y favorece el conjunto de actos violentos contra las niñas y las mujeres. 
Artículos científicos - Observatorio Universitario de Violencias contra las Mujeres

Contribuyen al feminicidio el silencio social, la desatención, la idea de que hay problemas más urgentes y la vergüenza y el enojo que no conminan a transformar las cosas sino a disminuir el hecho y demostrar que no son tantas "las muertas" ... (Lagarde, 2008: 217).

Así, siguiendo las argumentaciones de las autoras citadas, encontramos que existe una íntima confluencia de procesos, ámbitos, instituciones y actores que aceitan y se sirven de los mecanismos de opresión hacia las mujeres. Ello deriva en violencia de género, la cual se recrea de manera específica según sean los tipos y modalidades en que se ejerza. Una de ellas es la que constituye el foco de interés del presente artículo.

De acuerdo con Ana Isabel Blanco García (2005), la violencia doméstica puede definirse como

\begin{abstract}
un tipo concreto de violencia de género o contra las mujeres, que se manifiesta en una clase de comportamientos abusivos (físicos, sexuales, emocionales) que se dan en el contexto de una relación afectiva cercana, ya sea entre parejas casadas, que cohabitan o exparejas. No se limita a los miembros de la pareja (sea esta heterosexual $u$ homosexual), sino que a veces afecta también a los descendientes o a otros miembros de la familia... es un tipo de violencia contra las mujeres, ejercida por los varones, basándose en una posición de superioridad que les otorga una organización social concreta: la patriarcal. (Blanco, 2005: pp. 13-14).
\end{abstract}

Hay que resaltar la intimidad, emociones, vínculos y espacialidad compartida entre quienes circulan en ese ámbito, cuyo caos está regulado por el orden patriarcal. Esos elementos funcionan en la lógica del detrimento de las mujeres principalmente, aunque no únicamente: personas adultas mayores, niñas y niños, integrantes del ámbito doméstico con alguna situación específica de salud tanto física y/o mental, también son objeto de la violencia doméstica.

Sin embargo, en las siguientes páginas se desarrollará el término violencia doméstica en función de la especificidad que tiene cuando agrede y somete a las mujeres, entiéndase niñas, adultas y adultas mayores. No obviemos que este tema ha cobrado dimensiones alarmantes, por lo que se ha tenido que legislar en la materia. Ejemplos de ello son la Ley General de Acceso de las Mujeres a una Vida Libre de Violencia (LGAMVLV), vigente a nivel federal desde el 1 de febrero de 2007; y la Ley de Acceso de las Mujeres a una Vida Libre de Violencia para el estado de Veracruz de Ignacio de la Llave (LAMVLV), emitida en 28 de febrero de 2008. Esta última señala también que los tipos de violencia son entendidos como aquellos actos u omisiones que dañan la dignidad, la integridad y la libertad de las mujeres. (Gobierno del estado de Veracruz de Ignacio de la Llave, 2011. p. 5 


\section{Artículos Científicos - Observatorio Universitario de Violencias contra las Mujeres}

Para Veracruz establece que la violencia psicológica, ${ }^{2}$ física, ${ }^{3}$ sexual, ${ }^{4}$ patrimonial, ${ }^{5}$ económica ${ }^{6}$ y obstétrica ${ }^{7}$ son considerados tipos de violencia, así como cualquiera que lesionen o dañe la dignidad, integridad y libertad de las mujeres. (ibid)

Retomando la situación de que las nuevas tecnologías de la comunicación reformulan y reproducen estrategias para violentar a las mujeres, la LAMVLV de Veracruz también define a los tipos de violencia como:

\footnotetext{
${ }^{2}$ La define como "Acto u omisión que dañe la estabilidad psíquica y/o emocional de la mujer; consistente en amedrentar, negligencia, abandono, celotipia, insultos, humillaciones, denigración, marginación, infidelidad, comparaciones destructivas, rechazo y restricción a la autodeterminación". (Gobierno del estado de Veracruz de Ignacio de la Llave, 2011. p. 5.)

${ }^{3}$ La Ley señala que es el "Acto que inflige daño usando la fuerza física o algún tipo de arma u objeto que pueda provocar o no lesiones ya sean internas, externas, o ambas". (ibid)

${ }^{4}$ La Ley define a la violencia sexual como el "Acto que degrada o daña el cuerpo y/o la sexualidad de la víctima, que atenta contra su libertad, dignidad e integridad, como una expresión de abuso de poder que implica la supremacía sobre la mujer, al denigrarla o concebirla como objeto; se considera como tal, la discriminación o imposición vocacional, la regulación de la fecundidad o la inseminación artificial no consentidas, la prostitución forzada, la pornografía infantil, la trata de niñas y mujeres, la esclavitud sexual, el acceso carnal violento, las expresiones lascivas, el hostigamiento sexual, la violación, los tocamientos libidinosos sin consentimiento o la degradación de las mujeres en los medios de comunicación como objeto sexual". (ibid, p. 5 y 6)

${ }^{5}$ De acuerdo con la LAMVLV para el estado de Veracruz, la violencia patrimonial es el "Acto u omisión que afecta la supervivencia de la víctima; se manifiesta en la transformación, sustracción, destrucción, retención o distracción de objetos, documentos personales, bienes y valores, derechos patrimoniales o recursos económicos destinados a satisfacer sus necesidades y puede abarcar los daños a los bienes comunes o propios de la víctima". (ibid. p. 6)

${ }^{6}$ La violencia económica es definida en la Ley local como la "Acción u omisión de la persona agresora que afecta la supervivencia económica de la víctima; se manifiesta a través de limitaciones encaminadas a controlar el ingreso de sus percepciones económicas, asî como la percepción de un salario menor por igual trabajo, dentro de un mismo centro laboral; también se considerará como tal, el no reconocimiento de la paternidad y/o el incumplimiento de las obligaciones que se derivan de la misma". (ibid)

7 En tanto, la violencia obstétrica es definida por la LAMVLV como la "Apropiación del cuerpo y procesos reproductivos de las mujeres por personal de salud, que se expresa en un trato deshumanizador, en un abuso de medicalización y patologización de los procesos naturales, trayendo consigo pérdida de autonomía y capacidad de decidir libremente sobre sus cuerpos y sexualidad; se consideran como tal, omitir la atención oportuna y eficaz de las emergencias obstétricas, obligar a la mujer a parir en posición supina y con las piernas levantadas, existiendo los medios necesarios para la realización del parto vertical, obstaculizar el apego precoz del niño o niña con su madre sin causa médica justificada, negándole la posibilidad de cargarlo y amamantarlo inmediatamente después de nacer, alterar el proceso natural del parto de bajo riesgo, mediante el uso de técnicas de aceleración, sin obtener el consentimiento voluntario, expreso e informado de la mujer y practicar el parto por vía de cesárea existiendo condiciones para el parto natural, sin obtener el consentimiento voluntario, expreso e informado de la mujer". (ibid)
} 
Artículos científicos - Observatorio Universitario de Violencias contra las Mujeres

cualquier acto de violencia que se manifieste en acoso, hostigamiento, amenazas, insultos, violación de datos e información privada, divulgación de información apócrifa, mensajes de odio o la difusión, sin consentimiento de las mujeres, niñas o adolescentes, de contenido íntimo, textos, fotografías, videos, datos personales u otras impresiones gráficas o sonoras, verdaderas o alteradas, cometidos, instigados o agravados en parte o totalmente, y que se presenta a través de las Tecnologías de la Información y la Comunicación, plataformas de internet, redes sociales, sistemas de mensajería instantánea o correo electrónico, o cualquier otro espacio similar digitalizado; que atente contra la integridad, la dignidad, la intimidad, la libertad, la vida privada de las mujeres, y les cause un daño o sufrimiento psicológico, físico, económico o sexual, tanto en el ámbito privado, como en el público; así como daño moral a ellas o su familia... (ibid. p. 6)

Asimismo, la LAMVLV de Veracruz define a la modalidad de violencia como aquellas formas, manifestaciones y ámbitos en que se presenta la violencia contra mujeres. (ibid. p. 6 y 7) 


\section{Artículos Científicos - Observatorio Universitario de Violencias contra las Mujeres}

Dentro de ésta encontramos a la violencia de género, ${ }^{8}$ la que tiene lugar en el ámbito escolar, ${ }^{9}$ laboral ${ }^{10}$, en la comunidad, ${ }^{11}$ institucional, ${ }^{12}$ feminicida ${ }^{13}$ y política. ${ }^{14}$

Dentro de las modalidades o ámbitos en donde se agrede a las mujeres, define a la violencia en el ámbito familiar como:

${ }^{8}$ Definida en la Ley como "Cualquier acción u omisión, basada en el género... que les cause a las mujeres de cualquier edad, daño o sufrimiento psicológico, físico, patrimonial, económico, sexual o la muerte tanto en el ámbito privado como en el público y que se expresa en amenazas, agravios, maltrato, lesiones, y daños asociados a la exclusión, la subordinación, la discriminación y la explotación de las mujeres y que es consubstancial a la opresión de género en todas sus modalidades afectando sus derechos humanos. La violencia de género contra las mujeres involucra tanto a las personas como a la sociedad, comunidades, relaciones, prácticas e instituciones sociales, y al Estado que la reproduce al no garantizar la igualdad, al perpetuar formas legales, jurídicas, judiciales, políticas androcéntricas y de jerarquía de género y al no dar garantías de seguridad a las mujeres durante todo su ciclo de vida..." (ibid, p. 6 -7).

9 "Conductas que dañan la autoestima de las alumnas con actos de discriminación por su sexo, edad, condición social, condición étnica, condición académica, limitaciones y/o características físicas, que les infligen maestras o maestros, personal directivo, administrativo, técnico, de intendencia, o cualquier persona prestadora de servicios en las instituciones educativas...". (ibid)

10 "Acto u omisión en abuso de poder que daña la autoestima, salud, integridad, libertad y seguridad de la víctima, e impide su desarrollo y atenta contra la igualdad; constituye violencia laboral la negativa ilegal a contratar a la víctima o a respetar su permanencia o condiciones generales de trabajo; la descalificación del trabajo realizado, las amenazas, la intimidación, las humillaciones, la explotación, el impedimento a las mujeres de llevar a cabo el período de lactancia previsto en la Ley y todo tipo de discriminación por condición de género; se ejerce por las personas que tienen un vínculo laboral o análogo con la víctima, independientemente de la relación jerárquica". (ibid).

11 "Actos individuales o colectivos que transgreden derechos fundamentales de las mujeres y propician su degradación, discriminación, marginación o exclusión en el ámbito público”. (ibid)

12 “... actos $u$ omisiones de las y los servidores públicos... resultado de prejuicios de género, patrones estereotipados de comportamiento o prácticas sociales y culturales basadas en conceptos de inferioridad de las mujeres o de subordinación a los hombres, que discriminen o tengan como fin dilatar, obstaculizar, impedir el goce y ejercicio de los derechos humanos de las mujeres, así como su acceso al disfrute de políticas públicas destinadas a prevenir, atender, investigar, sancionar y erradicar los diferentes tipos de violencia". (ibid)

13 "Forma extrema de violencia de género contra las mujeres, producto de la violación de sus derechos humanos, en los ámbitos público y privado, conformada por el conjunto de conductas misóginas que pueden conllevar tolerancia social e indiferencia del Estado y puede culminar en homicidio y otras formas de muerte violenta de mujeres". (ibid)

14 “....acción u omisión, incluida la tolerancia, basada en elementos de género y ejercida dentro de la esfera pública o privada, que tenga por objeto o resultado limitar, anular o menoscabar el ejercicio efectivo de los derechos políticos y electorales de una o varias mujeres, el acceso al pleno ejercicio de las atribuciones inherentes a su cargo, labor o actividad; el libre desarrollo de la función pública, la toma de decisiones, la libertad de organización, así como el acceso y ejercicio a las prerrogativas, tratándose de precandidaturas, candidaturas, o su función en el ámbito público". (ibid. p. 8) 
Artículos científicos - Observatorio Universitario de Violencias contra las Mujeres

... acto abusivo de poder u omisión intencional, dirigido a dominar, someter, controlar, o agredir de manera física, verbal, psicológica, patrimonial, económica y sexual de las mujeres, dentro o fuera del domicilio familiar, ejercida por personas que tengan o hayan tenido relación o parentesco, concubinato o que mantengan o hayan mantenido una relación de hecho con la víctima. (2011: p. 7)

La violencia en el ámbito doméstico y/o familiar nos permite vislumbrar qué pasa en materia de feminicidio en el país. Constituye un espacio en donde poco se ha ahondado para conocer y analizar lo que ahí acontece en materia de violencia contra las mujeres, esto pese a que existe todo un andamiaje legal que sanciona los delitos perpetrados en los hogares.

\section{Violencia contra las mujeres en México. Cifras antes de la pandemia.}

En coincidencia con Castañeda, Ravelo y Pérez (2013), en el texto La violencia feminicida en México, aproximaciones y tendencias 1985-2016, de la Secretaría de Gobernación, el Instituto Nacional de las Mujeres y ONU Mujeres (2017), se establece que la discriminación y desigualdad de género contra las mujeres, se expresa en actos cotidianos y sistemáticos de violencia que se cometen contra ellas. La agresión es diaria y está presente en todos los ámbitos, por lo que constituye violaciones reiteradas a los derechos humanos de las mujeres, extendida y arraigada en el mundo. Sus esquirlas se incrustan en su vida, salud, libertad y seguridad. Mina el desarrollo de países y daña a la sociedad en su conjunto. (ibid, p. 10)

Una de las violencias letales es el feminicidio. Tal como lo señala la Antropóloga Feminista Marcela Lagarde (2008), se trata del genocidio contra mujeres; se perpetra cuando las condiciones históricas generan prácticas sociales que solapan atentados violentos contra la integridad, salud, libertades y la vida de las mujeres.

En el feminicidio concurren en tiempo y espacio, daños contra niñas y mujeres realizados por conocidos y desconocidos, por violentos, -en ocasiones violadores-, y asesinos individuales y grupales, ocasionales o profesionales, que conducen a la muerte cruel de algunas de las víctimas... No todos los crímenes son concertados o realizados por asesinos seriales: los hay seriales e individuales, algunos son cometidos por conocidos... Sin embargo, todos tienen en común que las mujeres son usables, prescindibles, maltratables y desechables...todos coinciden en su infinita crueldad y son, de hecho, crímenes de odio contra las mujeres... Para que se de el feminicidio concurren, de manera criminal, el silencio, la omisión, la negligencia y la colusión parcial o total de autoridades encargadas de prevenir y erradicar estos crímenes. Su ceguera de género o sus prejuicios sexistas y misóginos sobre las mujeres. (Lagarde, 2008: p. 216) 


\section{Artículos Científicos - Observatorio Universitario de Violencias contra las Mujeres}

La tipificación del feminicidio en México es reciente. Se incorporó al Código Penal Federal en 2012 y es definido como el delito a partir del cual se priva de la vida a una mujer por razones de género (Cámara de diputados del H. Congreso de la Unión, 2020). En el estado de Veracruz se tipificó en 2011 y define a este delito en los mismos términos en que lo hace el Código Federal (Congreso del estado de Veracruz, 2020): se señala que "Comete el delito de feminicidio quien por razones de género priva de la vida a una mujer". Dichas razones de género son las siguientes:

1. Exista o haya existido entre el activo y la víctima una relación de parentesco por consanguinidad o afinidad, de matrimonio, concubinato, noviazgo o cualquier otra relación de hecho o amistad;

2. Exista o haya existido entre el activo y la víctima una relación laboral, escolar, o cualquier otra que implique confianza, subordinación o superioridad;

2Bis. El activo se haya valido de su relación como conductor de un vehículo de transporte de pasajeros, turismo o cualquier otra modalidad;

3. La víctima presente signos de violencia sexual de cualquier tipo;

4. A la víctima se le hayan infligido lesiones infamantes, degradantes o mutilaciones previamente a la privación de la vida, o se realicen marcas infamantes o degradantes sobre el cadáver, o éste sea mutilado;

5. Hayan existido amenazas, acoso o lesiones del sujeto activo en contra de la víctima;

6. El cuerpo de la víctima sea expuesto o arrojado en un lugar público; o

7. La víctima haya sido incomunicada.

Fue a partir de 2015 que el Secretariado Ejecutivo del Sistema Nacional de Seguridad Pública (SESNSP) realizó reportes retomando en específico las carpetas iniciadas por el delito de feminicidio en el plano nacional. Sin embargo, previo a ello, se cuenta con documentos de carácter oficial que, si bien no retoman esta categoría reciente, documentan los homicidios de mujeres. Así tenemos que entre 1985 y 2016 se registraron 52,210 homicidios de mujeres en el país. De estos, el 29.8\% tuvieron lugar entre 2010 y 2016 (ibid, p.18). En este último año se registró el repunte de dicho delito: la tasa nacional de defunciones femeninas con presunción de homicidio (DFPH) fue de 4.4; exhibiendo el estado de Colima la más alta del país con 16.3, seguido por el estado de Guerrero, con 13.1. Por su parte, ese año Veracruz registró una tasa de 3.3; por debajo de la media nacional (SEGOB, Inmujeres y ONU Mujeres, 2017: p. 22).

La distribución porcentual de las DFPH que tuvieron lugar en 2016, indicó que entre los tres medios que provocaron los decesos destacó el arma de fuego con el $48.4 \%$ de los casos en donde mujeres perdieron la vida. El 17.2\% de los decesos se debió a ahorcamiento y el 16.6\% fue por objeto cortante. En el 73\% de los casos la agresión se realizó en el hogar (ibid, pp.39 y 42). 
De acuerdo con el documento La violencia feminicida en México, aproximaciones y tendencias 1985-2016, los grupos etarios más vulnerables para ser víctima de un asesinato en el hogar estuvieron constituidos en ese periodo por aquellas que se ubicaron entre los 10 y 14 años de edad; así como por las adultas mayores, particularmente aquellas que rebasaban los 70 años (ibid, p. 45).

Entre los años 2000 y 2015 se asesinaron a 28, 175 mujeres (Kánter, 2016: p. 21). De éstas 2,610 eran niñas y adolescentes. El 20\% eran menores de un año; el 28\% se ubicaban entre uno y 4 años. Del rango de 5 a 9 años constituyó el 19\% y 33\% estuvo conformado por menores que se ubicaban en el rango de 10 a 14 años.

Es importante mencionar que, en el año 2004, Marcela Lagarde encabezó el proyecto Investigación diagnóstica sobre violencia feminicida en la República Mexicana, basándose en datos oficiales. Contabilizó 1,205 niñas y mujeres asesinadas en el país tan solo en ese año. A partir de datos retomados de INEGI y Secretaría de Salud, concluyó que, en promedio, cada día de ese año cuatro niñas y mujeres fueron asesinadas.

En los primeros cinco años del siglo XXI eran asesinadas en México mil niñas y mujeres anualmente. En lo que corresponde al estado de Veracruz, en 2004, el equipo de trabajo de este proyecto encabezado por Lagarde registró 264 niñas y mujeres asesinadas, lo que significó una tasa de 1.144. (Lagarde, 2008)

En dicha entidad federativa, el 28 de septiembre de 2011, al ser requerido por la "Comisión especial para conocer y dar seguimiento puntual y exhaustivo a las acciones que han emprendido las autoridades competentes en relación a los feminicidios registrados en México", de la LXI Legislatura de la Cámara de Diputados, el entonces Procurador General de Justicia del estado de Veracruz, Reynaldo Escobar Pérez, informó que de 2000 a 2010 el número de investigaciones que se iniciaron por el probable delito de homicidio doloso contra mujeres fue de 728 casos (Escobar, 2011). Es decir, en promedio 73 veracruzanas fueron asesinadas cada año a lo largo de esa década.

Informó a la Comisión especial que, en la década referida, los medios utilizados para asesinar a las veracruzanas fueron los siguientes: 
Tabla 1.

Medio con el que se privó de la vida a las mujeres. Periodo 2000 -2010.

\begin{tabular}{|c|r|}
\hline Medio con el que se privó de la vida & Total \\
\hline Arma blanca o punzo cortante & 201 \\
\hline Arma de fuego & 165 \\
\hline Golpes & 128 \\
\hline Estrangulada & 65 \\
\hline Golpes y violación & 11 \\
\hline Calcinada & 7 \\
\hline Ahogada & 4 \\
\hline Descuartizada & 2 \\
\hline Envenenada & 2 \\
\hline Degollada & 1 \\
\hline No especificado & 142 \\
\hline Total & 728 \\
\hline
\end{tabular}

Fuente Escobar (2011).

De acuerdo a los datos que presentó el funcionario, la vivienda y la vía pública fueron los lugares en donde principalmente se localizaron los 728 cuerpos sin vida. Se estableció, a partir de las averiguaciones, que en la mayoría de los casos (601 del total) no se tenía noticia sobre quién había cometido el feminicidio, tal como se presenta en la tabla 2.

Tabla 2.

Medio con el que se privó de la vida a las mujeres. Periodo 2000 -2010.

\begin{tabular}{|c|c|}
\hline Relación con el victimario & Total \\
\hline Esposo o concubino & 59 \\
\hline Por consanguinidad & 36 \\
\hline Ninguna & 19 \\
\hline Amistad & 10 \\
\hline Expareja & 3 \\
\hline No especificado & 601 \\
\hline Total & $\mathbf{7 2 8}$ \\
\hline
\end{tabular}

Fuente Escobar (2011).

Es importante destacar que el informe emitido por Escobar Pérez constituye la única información oficial que se hizo pública por parte de la antigua Procuraduría estatal en ese periodo gubernamental y en los subsecuentes. 
Por otra parte, regresando al plano nacional, en lo correspondiente a 2018, INEGI (2019) informó que 30 de cada 100 mujeres asesinadas en ese año fue estrangulada, ahorcada o sofocada, ahogada, quemada, golpeada con algún objeto o por herida con arma punzocortante. Esto nos habla de una ventaja de proximidad del agresor para someter, torturar y acabar con la vida de la víctima.

De acuerdo con datos de la Dirección General de Información en Salud, en 2019 cada día 279 mujeres recibieron atención médica por lesiones a causa de violencia familiar en su vivienda. En ese mismo año el Secretariado Ejecutivo del Sistema Nacional de Seguridad Pública (SESNSP) registró 945 víctimas de feminicidio. Al contabilizar el registro que el Secretariado posee desde 2015, hace un total de 3,593 feminicidios en cinco años.

La violencia contra las mujeres en México ha tenido un grave repunte en la última década y ha dejado secuelas devastadoras en la cotidianidad de aquellas mujeres que han sobrevivido y que continúan siendo objeto de violencia. En ello ha influido fuertemente el crimen organizado (narcotráfico, tráfico de armas y tráfico de mujeres para trabajo sexual forzado),

pero debemos observar que los asesinatos, desapariciones y agresiones cometidas contra mujeres desde tiempo atrás han venido realizándose con la permisividad social y gubernamental que caracteriza al país.

El Instituto Nacional de Estadística y Geografía (INEGI), a partir de la socialización de los resultados de la Encuesta Nacional sobre la Dinámica de las Relaciones en los Hogares (ENDIREH) 2016, señaló que el 43.9\% de las mexicanas de 15 años y más, enfrentaron agresiones de su esposo o pareja. Un dato que no pasa desapercibido en ese informe es que el $40 \%$ de los homicidios de mujeres fueron cometidos por sus parejas en los hogares que compartían (INEGI, 2017).

Esos escenarios fatales se van construyendo a través de los años: el 66.1\% de las mexicanas de 15 años y más han experimentado por lo menos un acto de violencia por parte de distintos agresores: pareja, familiar, compañero de escuela o del trabajo, amistades o desconocidos.

Todo ello se traduce en que 30.7 millones de mujeres en México han sido objeto de actos de violencia y discriminación a lo largo de su vida (INEGI, 2019). 


\section{Artículos Científicos - Observatorio Universitario de Violencias contra las Mujeres}

Las mujeres que están más expuestas a la violencia de pareja son las que se encuentran entre los 20 y 39 años de edad, pues el 70\% enfrentó por lo menos algún episodio de violencia o abuso (ibid).

\section{Violencia contra las mujeres en medio de la contingencia sanitaria. ¿Pandemia silente?}

Durante la contingencia sanitaria cuyo inicio fue marcado con la apertura de la Jornada Nacional de Sana Distancia, la violencia de género contra las mujeres recibió un fuerte impulso gracias a tres elementos omnipresentes en 2020: el confinamiento domiciliario, la crisis económica derivada de la suspensión de actividades no esenciales y los padecimientos provocados en miles de familias del país por el coronavirus.

Instancias gubernamentales de los tres órdenes de gobierno, organizaciones de la sociedad civil y de la academia generaron información que hizo evidente este efecto colateral de la pandemia. Los datos generados permitieron que el tema de la violencia doméstica constituyera uno de los principales debates nacionales que tuvieron lugar en el primer semestre de 2020. También obligaron a que diferentes actores y actoras del país, funcionariado de primer nivel y las instancias que atienen lo correspondiente, se posicionaran sobre el tema y tomaran medidas sobre el asunto; aunque lamentablemente fue un esfuerzo acotado e insuficiente ante la grave dimensión del problema, los recortes presupuestales y las medidas de austeridad que afectaron en gran medida a la protección de la vida y los derechos de la población en general y particularmente a las mujeres.

De acuerdo con información generada por el SESNSP (2020), de enero a junio de 2020 en México se cometieron 473 feminicidios; 62 más de los que tuvieron lugar durante el año 2015, por ejemplo. El estado de Veracruz ocupa el segundo sitio a nivel nacional con 45 casos, solo antecedido por el Estado de México con 63. El Secretariado ubica al municipio de Veracruz en el décimo quinto lugar de la lista de los municipios con más casos de feminicidios en México. Coatepec y Xalapa se encuentran los sitios 39 y 40. Gutiérrez Zamora en el número 84, Lerdo de Tejada en el 85, Papantla en el 86, San Andrés Tuxtla y Tihuatlán en los lugares 87 y 88 , respectivamente. (ibid)

Un tema que tiene íntima relación con el feminicidio es el homicidio de mujeres. De acuerdo con datos oficiales generados también por el Secretariado, de enero a junio de este año se perpetraron 1,443 homicidios dolosos de mujeres y 1490 homicidios culposos de mujeres. Para ambos delitos, el estado de Guanajuato ocupa el primer sitio con 252 asesinatos y 326, respectivamente. (ibid) 
Retomando los registros realizados en el informe sobre violencia contra las mujeres que mensualmente da a conocer el SESNSP, observamos que en los meses posteriores al inicio de la Jornada Nacional de Sana Distancia, su conclusión y la "nueva normalidad", es decir en abril, mayo y junio, tuvieron lugar 233 feminicidios. En este mismo periodo, el estado de Veracruz registró 20 delitos de este tipo.

En materia de homicidios dolosos contra mujeres ocurridos en los meses citados anteriormente, el SESNSP reportó 723. En el estado de Veracruz se contaron un total de 25. En lo referente a víctimas mujeres de homicidios culposos, en el periodo citado se registraron 857 a nivel nacional, ejecutándose 41 en la entidad veracruzana.

Si retomamos el total de mujeres asesinadas en el país durante los tres primeros meses de la contingencia sanitaria (abril, mayo y junio), suman 1,589 decesos. En Veracruz la cifra asciende a 86. Independientemente de que los ilícitos (feminicidios y homicidios de mujeres) se realizaran en el ámbito doméstico o en el público, lo cierto es que estas cifras generadas por el gobierno federal nos permiten vislumbrar la peligrosidad que este país y la entidad veracruzana guardan para la vida y seguridad de las ciudadanas de cualquier edad.

En cuanto a los datos generados desde la sociedad civil, principalmente desde organizaciones feministas, la Red Nacional de Refugios ${ }^{15}$ reportó que, durante los primeros dos meses de la Jornada Nacional de Sana Distancia, concretamente entre marzo y mayo de 2020, brindó atención a 12,710 mujeres, niñas y niños a través de llamadas de auxilio que recibió por parte de las víctimas que acudían a la Red en busca de ayuda. Atendieron directamente a 6, 978 mujeres y sus hijos e hijas, lo que implicó el incremento del 77\% en comparación del mismo periodo en 2019. Un dato para destacar es que más de la mitad de los refugios del país han tenido un incremento del 50\% en los ingresos de mujeres, niños y niñas (Intersecta, Equis Justicia para las Mujeres y La Red Nacional de Refugios, 2020).

El 100\% de las mujeres que ingresaron a los espacios de atención y resguardo coordinados por la Red fueron víctimas de violencia familiar y violencia psicológica, 49.47\% fue objeto de violencia física, 43.37\% de violencia económica, 25.95\% de violencia patrimonial, 17.62\% de violencia sexual y el $4 \%$ sufrieron intentos de feminicidio. El 79\% de las mujeres que lograron

\footnotetext{
${ }^{15}$ La Red se encuentra integrada por 69 espacios de prevención, atención y protección para mujeres, niños y niñas que viven violencia. Está conformada por 31 Refugios, 29 Centros de Atención Externa, cinco Casas de Emergencia y cuatro Casas de Transición distribuidos en los 32 estados de país.
} 


\section{Artículos Científicos - Observatorio Universitario de Violencias contra las Mujeres}

ingresar a los refugios en abril de 2020 habían sido víctimas de violencia emocional, económica y física. Por otra parte, las niñas y niños que las acompañaban también fueron afectados por los eventos violentos acontecidos durante el confinamiento: el 5\% fueron víctimas de abuso sexual y el $48 \%$ recibieron agresiones en sus hogares. (ibid)

En medio de este panorama, las mujeres que guardan una situación de mayor vulnerabilidad son aquellas que concentran en su cuerpo y su vida una vivencia de la violencia atravesada por su origen étnico, empobrecimiento y marginalidad. Así tenemos que más de 6 millones de mujeres pertenecen a alguno de los 68 pueblos indígenas del país. Su pertenencia étnica hace que vivan de manera específica la violencia en los ámbitos doméstico y público. A esto se suman otros elementos contextuales que complejizan vivir la pandemia en condiciones de marginalidad. El 23 de abril el presidente de la república emitió un decreto que establece medidas de austeridad para reducir el gasto público federal para hacer frente a la pandemia. Establece que no se ejercerá el 75\% del presupuesto disponible en las partidas de servicios generales y materiales y suministros (DOF, 2020). Dicho decreto presidencial destaca 38 programas prioritarios que no se verían afectados, pero no consideró a ningún programa relativo a la protección de las mujeres en situación de violencia, la debida ejecución de las declaratorias de Alerta de Violencia de Género contra las Mujeres que se ciernen en más de la mitad de las entidades federativas del país. Tampoco consideró a programa alguno que se ejecutara con perspectiva de género.

Si bien es cierto que no fueron afectados los presupuestos correspondientes al Fondo para el Bienestar y el Avance de las Mujeres (FOBAM) y el Programa de Fortalecimiento a la Transversalidad de la Perspectiva de Género (PFTPG), éstos demoraron en otorgarse aún más que de costumbre ya que la Secretaría de Hacienda demoró en ministrar los recursos. La situación afectó visiblemente a las instancias municipales de las mujeres (o institutos municipales), ya que aún en el mes de agosto de 2020, al menos en el estado de Veracruz no contaban con el recurso en los institutos municipales.

Entre los programas importantes que se afectaron porque no se consideraron prioritarios, fueron los de la Casa de la Mujer Indígena y Afromexicana, conocida popularmente como CAMI. Esto perjudicó directamente a la población de 35 regiones indígenas de México, pues las CAMI apoyan a las mujeres de los pueblos originarios a enfrentar la violencia con mayor certeza y en muchos casos dan acompañamiento para que denuncien ante las autoridades. Ante la presión de organizaciones de la sociedad civil y de entidades internacionales, les fue prometida la ministración de recursos que constituían el 50\% del presupuesto original que les permite la atención de usuarias y desplazamiento de las promotoras indígenas para realizar sus funciones (Red Nacional de Casas de las Mujeres Indígenas, 14 de julio de 2020). 
Artículos científicos - Observatorio Universitario de Violencias contra las Mujeres

En el marco de estos recortes y la afectación a la vida y seguridad de las mujeres del país, el 15 de julio de 2020 la Junta de Gobierno del Instituto Nacional de las Mujeres (Inmujeres) aprobó por mayoría de votos la reducción presupuestal del 75\% a esta instancia: más de 151 millones de pesos de los rubros de servicios generales, materiales y suministros. Si bien lo programas de atención a las mujeres no fueron tocados por estos recortes, lo cierto es que esta afectación marcó un retroceso que afecta la operatividad del Instituto en las importantes tareas que debe atender. ${ }^{16}$

Por ejemplo, y de acuerdo con datos referidos por la titular del Inmujeres, Nadine Gasman Zylbermann, el embarazo adolescente está generando un costo para el país que asciende a 63 mil millones de pesos. Dicha cifra es el equivalente al 0.25 del Producto Interno Bruto (PIB). Las adolescentes carecen de educación sexual integral y acceso a los servicios para la salud sexual, tampoco hay que olvidar que son víctimas de violencia sexual y pederastia. Cada año hay 380 mil madres adolescentes en México y diariamente son madres 32 niñas que regularmente han sido violadas y, por tanto, víctimas de pederastia (Canal Once, 10 de agosto de 2020).

\section{Violencias contra las mujeres en Veracruz en tiempos de Covid}

En este contexto complejo que merma los derechos humanos de las mujeres en el país, ¿qué sabemos de la violencia doméstica que padecen las veracruzanas? ¿Qué sucede en los municipios veracruzanos a partir del inicio del distanciamiento social y la "nueva normalidad" generadas por el SARS-CoV-2?

No es frecuente que los municipios del país manejen datos abiertos que den certeza informativa a la ciudadanía en lo referente a temas sensibles. En este sentido, observamos que los gobiernos municipales de la entidad no cuentan con este mecanismo de rendición de cuentas. Pese a ello, localizamos que el ayuntamiento del municipio de Xalapa, capital del estado de Veracruz, es la excepción a esta situación. En su portal de internet da a conocer informes periódicos sobre la violencia que enfrentan las mujeres que habitan esa demarcación municipal. Dicha información es generada por el Instituto Municipal de las Mujeres de Xalapa (IMMX).

\footnotetext{
${ }^{16}$ La información apareció en diversos medios de comunicación nacionales. Tuve oportunidad de conocer los datos de primera mano debido a que fueron materia de la Segunda Sesión Ordinaria de la Junta de Gobierno del Instituto Nacional de las Mujeres (Inmujeres), de la cual formo parte.
} 


\section{Artículos Científicos - Observatorio Universitario de Violencias contra las Mujeres}

Esta instancia municipal cuenta con dos mecanismos de atención que le permiten documentar la violencia que viven las ciudadanas. Uno de ellos es el Departamento de Atención a la Violencia, el cual ofrece atención de manera presencial a las que así lo requieran. Otro mecanismo es la Línea Violeta, que vía telefónica ofrece atención psicológica y legal a mujeres víctimas de violencia.

En el documento "Incidencias de violencia de género contra las mujeres reportadas al Instituto Municipal de las Mujeres de Xalapa (IMMX). Periodo de contingencia sanitaria COVID19. Reporte global. Del 16 de marzo al 02 de agosto de 2020", informaron que existía un aumento del 57\% en las llamadas recibidas a la Línea Violeta Xalapa, en comparación a las mismas semanas de 2019. Las atenciones de carácter presencial aumentaron en un 18\% en comparación al mismo periodo de 2019. (IMMX, 2020)

De acuerdo con sus registros, el IMMX detectó un aumento del 12\% en la violencia psicológica, 10\% en la violencia sexual y 55\% en violencia patrimonial. Además, la violencia en el ámbito doméstico aumentó 180\%. Asimismo, detectaron disminución del 36\% del consumo de alcohol como detonante de las agresiones en contra de las víctimas de violencia de género. (ibid) Este último punto se derivó de la iniciativa del gobierno municipal para regular la venta de alcohol en el municipio con el fin de inhibir uno de los detonantes de violencia más frecuentes.

Sin embargo, cabe señalar que el IMMX informó en el reporte mencionado que el uso de dogas ilegales en situaciones de violencia contra las mujeres en el municipio se incrementó en un 31\% en comparación con el mismo periodo en 2019.

En su conjunto, esta información generada por el municipio de Xalapa es sumamente valiosa. Si bien la población femenina en esa demarcación municipal asciende a 254 mil 295 y el número de mujeres atendidas por el IMMX en el periodo de contingencia fue de 629 personas, ${ }^{17}$ sus registros permiten conocer de manera aproximada sobre los principales tipos de violencia que viven las usuarias y el contexto en donde se realizan.

Aunque de manera parcial, el gobierno municipal puede medir el impacto de los mandatos del Cabildo, como es el caso de la regulación de la venta del alcohol. Lamentablemente, es difícil contar con información de este tipo en los 211 municipios restantes de la entidad. Ello no nos permite conocer y visibilizar lo que está pasando en Veracruz en materia de violencia contra las mujeres y, de manera puntual, sobre violencia doméstica.

\footnotetext{
${ }^{17}$ Tanto el Departamento de Atención a la Violencia como la Línea Violeta de Xalapa reciben usuarias de otros municipios veracruzanos y estados de la República, ya que acuden en busca de apoyo. Aunque con menor frecuencia, también han acudido hombres en busca de asesoría.
} 
En este sentido, durante 2020 el Observatorio Universitario de Violencias contra las Mujeres (OUVMujeres) ha enfocado su trabajo de documentación y análisis de información emitida por medios de comunicación locales, para registrar lo que acontece en Veracruz sobre la violencia doméstica. Al hacer una revisión de 46 medios informativos hemos obtenido los datos que se desarrollan a continuación.

En el primer semestre de 2020 documentamos 301 agresiones hacia mujeres. Marzo presentó 70 casos, por lo que fue el mes con más eventos de este tipo. Acayucan fue el municipio en donde se presentaron más agresiones, con 41 registros, seguido por Veracruz con 36 y San Andrés Tuxtla con 29. Las violencias psicológica y física fueron las más recurrentes; se presentaron de manera combinada en 142 ocasiones. De las 301 agresiones, en 183 de éstas se trató de víctimas adultas, en 55 casos fueron menores de edad y cuatro adultas mayores.

En 217 eventos los agresores fueron hombres que actuaban en solitario. En ocho casos quien agredió fue mujer; 54 agresiones fueron grupales y se trataba de hombres y mujeres. En 22 notas informativas que consultamos, no se especificaba el sexo de la persona agresora.

En cuanto a la modalidad de las violencias, sobresalieron la comunitaria con 144 casos y la doméstica con 123. La modalidad de género y feminicida presentaron 12 casos cada uno; la institucional dos y la escolar cinco.

En lo que se refiere a la violencia doméstica, durante este semestre presentó el siguiente comportamiento mensual: en enero se registraron 15 eventos en el ámbito doméstico; febrero registró 21; marzo 25; abril 20; mayo destacó al presentar 32 casos; y en la recta final del periodo, en el mes de junio, encontramos 10.

En lo que se refiere a los tipos de violencia presentados en el ámbito doméstico, detectamos que las violencias psicológica y física contabilizaron 84 casos, siendo las más reportadas por las mujeres agredidas. También destaca la violencia económica con 12 reportes; la violencia psicológica con 7 casos. 
Gráfica 1. Modalidad de la violencia.

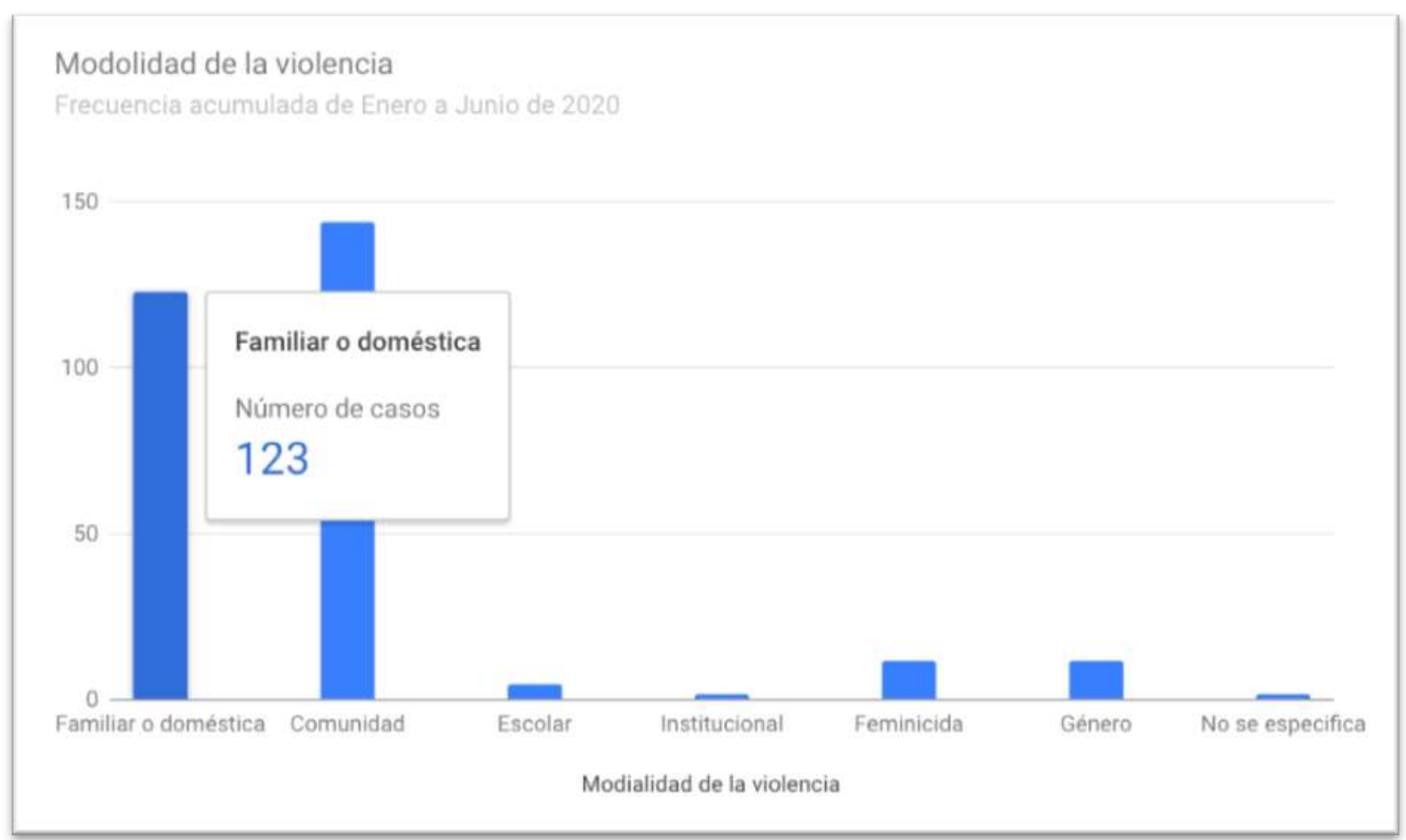

Elaboró: CUO, 2020.

Gráfica 2. Casos de violencia familiar o doméstica por mes.

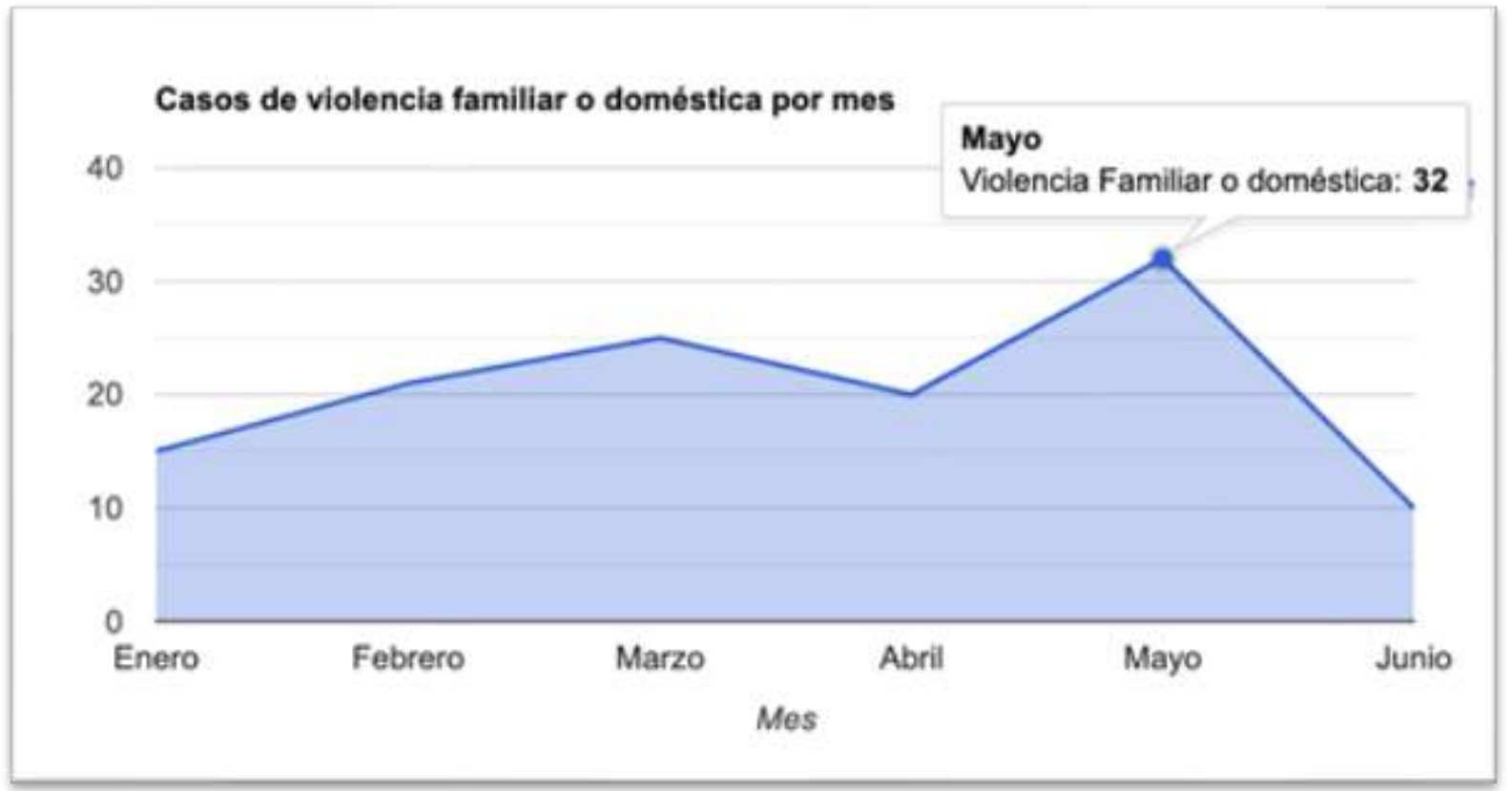

Elaboró: CUO, 2020.

Gráfica 3. Combinaciones de tipos de violencia familiar o doméstica. 


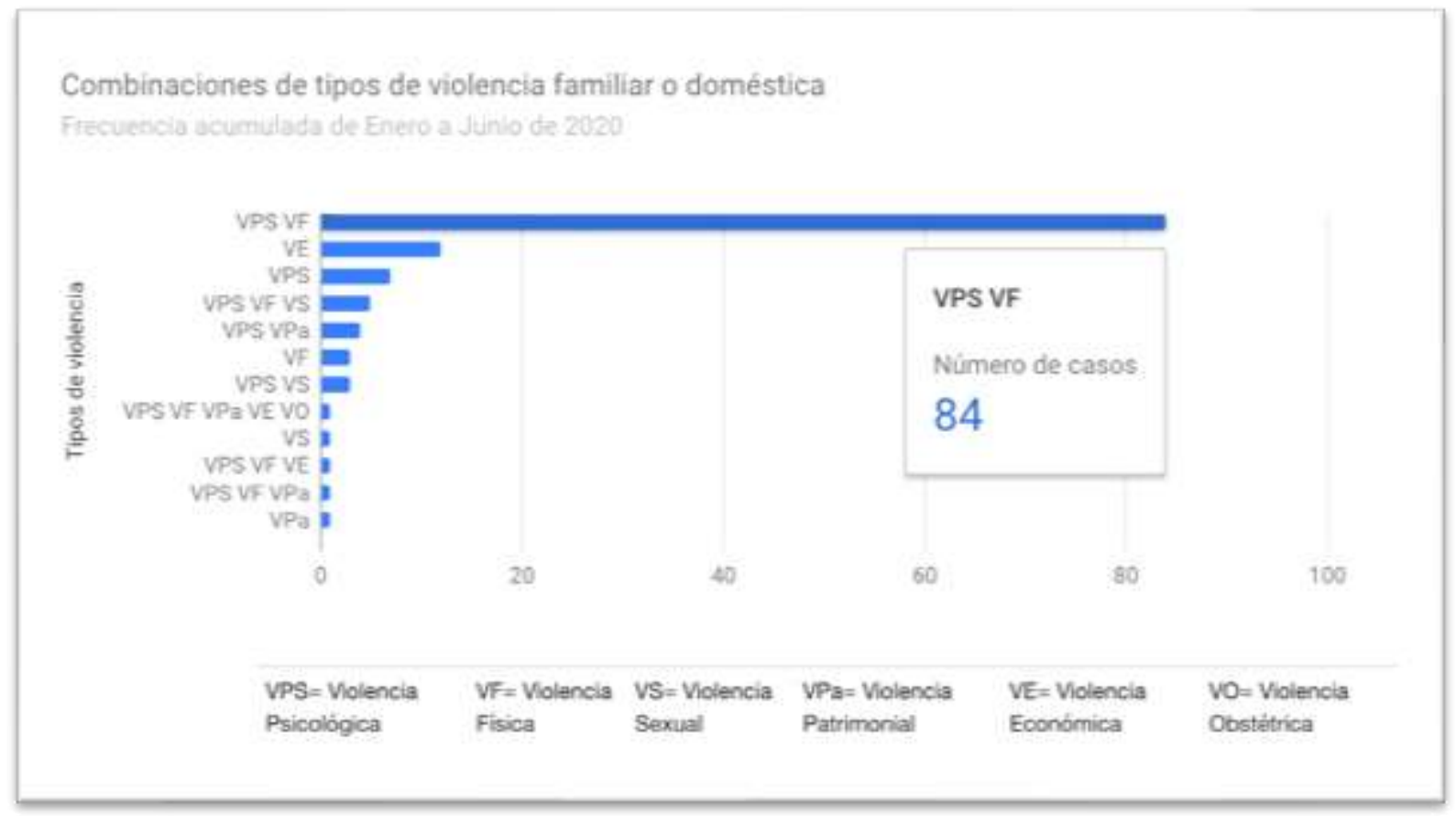

Elaboró: CUO, 2020.

Si bien estas son estimaciones numéricas obtenidas de los medios informativos, nos permiten vislumbrar numéricamente lo acontecido en el ámbito estatal; principalmente en el contexto de desmantelamiento de opciones para guarecerse de la violencia en el hogar.

\section{Conclusiones}

¿Por qué se señala que la violencia en el ámbito doméstico es una pandemia silente, a pesar de los datos que desde hace varios años nos dan elementos para evidenciar este fenómeno social de suyo grave?

La construcción de mecanismos de sujeción sobre las mujeres se engarza de tal manera con las violencias que logra invisibilizar, denostar y naturalizarlas. Si bien constituyen un grave problema de nuestros tiempos, logra pasar "desapercibido" gracias a la descalificación simbólica y cultural. Por ejemplo, las autoridades de mayor rango desestiman la gravedad del asunto y censuran la denuncia en torno al problema; por otro lado, gracias a diversas vías de comunicación se transmite un mensaje de odio y denostación al tema de las violencias contra las mujeres por parte de la sociedad en su conjunto, ello a pesar de la denuncia de diversos sectores. 
Tal como se ha señalado en las páginas de este documento, la violencia contra las mujeres, principalmente aquella que se presenta en el ámbito doméstico, continúa fortalecida en el marco de la contingencia sanitaria. El contexto, de por sí adverso, no puede ser más desalentador: la austeridad marcada desde el gobierno federal constituye una afectación directa a las ciudadanas del país, pero sobre todo es un grave retroceso para el ejercicio de los derechos de las mujeres de la República.

La merma de los programas y proyectos gubernamentales para fortalecer los derechos humanos de las mujeres y prestarles auxilio en el tema de violencia, está construyendo situaciones que en el corto plazo nos ha tocado observar, y cuyas secuelas mermarán la calidad de vida de la población femenina y la sociedad en general. Por ejemplo, las niñas y niños afectados por las esquirlas de la violencia doméstica están siendo educados sobre la definición convulsa que define a hombres y mujeres, las relaciones de poder que se tejen al interior de la esfera doméstica y el efectivo ejercicio de la violencia contra las mujeres.

Asimismo, se está generando un grave precedente en la definición legal del destino y uso presupuestal, la interferencia de decisiones coyunturales y la redirección que guardan estos fondos.

Este es el contexto en el que se desarrolla la violencia doméstica. Siempre bajo un ambiente político, social y gubernamental adverso que adereza su complejidad y la gravedad que va tomando a lo largo del tiempo. Ya observaremos qué giro toma la dinámica de las relaciones violentas en los hogares, así como el posible desarrollo de estrategias para abatirla. Será desde la sociedad civil feminista que se encabecen estas acciones. No podrá venir desde otro lado.

\section{Referencias}

Blanco García, A. I. (2005). Violencia doméstica y nuevos modelos de mujer: te doy mis ojos o la importancia de la mirada femenina. En Cuestiones de Género. Núm. 0, pp. 9-32.

Cámara de diputados del H. Congreso de la Unión (2020). Código penal federal. Ciudad de México: Cámara de diputados del H. Congreso de la Unión. Recuperado de

http://www.diputados.gob.mx/LeyesBiblio/pdf_mov/Codigo_Penal_Federal.pdf

Canal Once (10 de agosto de 2020). De buena fe: la agenda feminista en la 4T [Archivo de video]. Recuperado de https://www.youtube.com/watch?v=ohNm-MooWJE\&t=783s

Castañeda Salgado, M. P., Patricia Ravelo Blancas y Teresa Pérez Vázquez (2013). Feminicidio y violencia de género en México: omisiones del Estado y exigencia civil de justicia. En Iztapalapa. Revista de Ciencias Sociales y Humanidades. Núm. 74. Año 34. Enero-junio, pp. 11-39. 
Artículos científicos - Observatorio Universitario de Violencias contra las Mujeres

Congreso del estado de Veracruz (2020). Código penal para el estado de Veracruz de Ignacio de la Llave. Xalapa: Congreso del estado de Veracruz. Recuperado de:

https://www.legisver.gob.mx/leyes/LeyesPDF/CPENAL03072020.pdf

Diario Oficial de la Federación (DOF) (2020). Decreto por el que se establecen las medidas de austeridad que deberán observar las dependencias y entidades de la Administración Pública Federal bajo los criterios que en el mismo se indican. CDMX: Secretaría de Gobernación. Recuperado de https://dof.gob.mx/nota_detalle.php?codigo=5592205\&fecha=23/04/2020

Escobar Pérez, Reynaldo Gaudencio (2011). Comparecencia, Xalapa. Recuperado de http://www3.diputados.gob.mx/camara/001_diputados/010_comisioneslxi/002_especiales/0 08_feminicidios/08_reuniones_con_procuradores_locales/12_veracruz

Gobierno del estado de Veracruz de Ignacio de la Llave (2011). Ley de Acceso de las Mujeres a una Vida Libre de Violencia para el estado de Veracruz de Ignacio de la Llave. Xalapa: Instituto Veracruzano de las Mujeres.

Instituto Municipal de las Mujeres de Xalapa (IMMX) (2020). Incidencias de violencia de género contra las mujeres reportadas al Instituto Municipal de las Mujeres de Xalapa (IMMX). Periodo de contingencia sanitaria COVID-19. Reporte global. Del 16 de marzo al 02 de agosto de 2020. Xalapa: H. Ayuntamiento del Municipio de Xalapa.

Instituto Nacional de Estadística y Geografía (INEGI) (2019). Estadísticas a propósito del día internacional de la eliminación de la violencia contra la mujer (25 de noviembre). Datos Nacionales. Comunicado de prensa núm. 592/19

Instituto Nacional de Estadística y Geografía (INEGI) (2017). Resultados de la encuesta nacional sobre dinámica de las relaciones en los hogares (ENDIREH) 2016. Boletín de prensa núm. 379/17

Intersecta, Equis Justicia para las Mujeres y La Red Nacional de Refugios, AC (2020). Las dos pandemias. Violencia contra las mujeres en México en el contexto de COVID-19. CDMX: Intersecta, Equis Justicia para las Mujeres y La Red Nacional de Refugios, AC.

Kánter Coronel, Irma del Rosario (2016). Asesinatos de Mujeres en México. Ciudad de México: Senado de la República. LXIII Legislatura.

Lagarde y de Los Ríos, Marcela (2008). “Antropología, feminismo y política: violencia feminicida y derechos humanos de las mujeres. En Margaret Bullen y María Carmen Díez Mintegui (Coordinadoras). Retos teóricos y nuevas prácticas. Serie, XI Congreso de Antropología de la FAAEE. Donostia: Ankulegi Antropología Elkartea.

Red Nacional de Casas de las las Mujeres Indígenas (14 de julio de 2020). Boletín de la Red CAMI (página de Facebook). Recuperado el 5 de octubre de 2020.

https://www.facebook.com/Red-Nacional-De-Casas-De-Las-Mujeres-Indigenas-Camis355435571267495/?ref=page_internal 
Secretaría de Gobernación, el Instituto Nacional de las Mujeres y ONU Mujeres (2017). La violencia feminicida en México, aproximaciones y tendencias 1985-2016. México: SEGOB, Inmujeres, ONU Mujeres.

Secretariado Ejecutivo del Sistema Nacional de Seguridad Pública (SESNSP) (2020). Información sobre violencia contra las mujeres. Incidencia delictiva y llamadas de emergencia 911. Información con corte al 30 de junio de 2020. CDMX: Secretaría de Seguridad y Protección Ciudadana. Recuperado de https://drive.google.com/file/d/1wG6qya31zzz4m1YUgowZWSSHOz748HDt/view 